\title{
THE SIDE EFFECTS OF CHEMOTHERAPY IN GLIOMA
}

\author{
Neila Raisa ${ }^{1}$, Eko Arisetijono Marhaendraputro ${ }^{1}$ \\ Correspondence: neilaraisa@gmail.com \\ ${ }^{I}$ Department of Neurology Faculty of Medicine Brawijaya University, Malang, Indonesia.
}

\section{Article History: \\ Received: April 28, 2017 \\ Accepted: July 18, 2018 \\ Published: July 1, 2019}

Cite this as:

Raisa N, Marhaendraputro EA.

The side effects of chemotherapy

in glioma. Malang Neurology

Journal; 2019.5:92-97. DOI:

http://dx.doi.org/10.21776/ub.mnj

.2019.005.2.9

\section{ABSTRACT}

The increasing number of people who survive in the long term because of the advanced of glioma therapy with chemotherapy causes more slowly emerging neurologic side effects. Currently, there were three medical treatments are approved by the FDA for the management of glioblastoma namely temozolomide, carmustine wafer, and bevacizumab. However, most of the traditional chemotherapy drugs have a target in cell proliferation and cause toxicity of healthy cells. The side effects of chemotherapy on central nervous systems are chemobrain, acute encephalopathy, leukoencephalopathy, cerebellar dysfunction, and spinal cord toxicity. In addition, severe chemotherapy side effects may also occur in the peripheral nervous system called Chemotherapyinduced peripheral neuropathic pain (CIPNP). The clinician should recognize the symptoms of those side effect in glioma patients who received chemotherapy.

Keywords: Chemobrain, chemotherapy, CIPNP, glioma

\section{Introduction}

Glioma is defined as all tumors which are originated from stromal cells (glial) categorized as the astrocytic tumor (WHO classification grade I, II (astrocytoma), III (anaplastic), and IV (glioblastoma/GM), oligodendroglioma, ependymoma, and mixed glioma. ${ }^{1,2}$ Malignant glioma accounted for $75 \&$ of an overall primary brain tumor. ${ }^{3}$ More than $50 \%$ malignant glioma is glioblastoma (GBM) which is known as a most aggressive subtype. Patient with glioma had a poor prognosis (2-year survival rate for glioblastoma multiforme was ranged from $30,4 \%$ in $20-44$ years old patient until $1,3 \%$ in 75 years old patient or more), while 5-year survival rate was less than $5 \%$. $^{3,4}$

The role of chemotherapy as an essential step for management of glioma in the context of scientific development in last several years was increased because of two reasons: sensitivity of $1 \mathrm{p} / 19 \mathrm{q}$ on oligodendroglioma toward chemotherapy and availability of temozolomide, novel alkylating agents which has good penetration on the central nervous system. ${ }^{5}$ Relatively significant differences on the survival rate of a patient with glioblastoma multiforme after diagnosis were correlated with administration of chemotherapeutic agent Temodar (temozolomide) that could increase average survival rate in combined treatment (radiation therapy and chemotherapy) for 2.5 months compared to radiation therapy alone $(14,6$ months vs. 12,1 months, respectively). Furthermore, at the end of the second year, the survival rate in the radiotherapi+chemotherapy group was higher as compared to radiotherapy alone (26,5\% vs. $10,4 \%$, respectively).

Almost all conventional chemotherapeutic agent modulates the cell proliferation. Thereby, these drugs could affect normal cell and cause toxic effect because of its interference on the cell proliferation process. ${ }^{6}$ As the number of survivor increase because of development on cancer treatment, late neurological adverse drug reactions were highly found in this patient. ${ }^{3}$

\section{Chemotherapy for Glioma}

In 1990, phase II study demonstrated that temozolomide (TMZ) had a therapeutic effect on anaplastic glioblastoma and astrocytoma thus in $1999 \mathrm{TMZ}$ substituted nitrosourea as a chemotherapeutic agent for glioma. The previous study revealed that TMZ administration combined with radiotherapy has good efficacy for a patient with glioblastoma multiforme. ${ }^{5}$

\section{Temozolomide as Treatment for Glioma}

Recently, there is three available FDA-approved medicine for the treatment of GBM: temozolomide, wafer carmustine, and bevacizumab. Temozolomide/ TMZ is an oral preparation of alkylating agent derived from dacarbazine. TMZ is absorbed rapidly (peak concentration within 30-90 minutes) and hydrolyzed spontaneously at physiologic $\mathrm{pH}$ become active metabolite 5-(3-methyl-1thiazeno) imidazole-4-carboxamide (MTIC). Cytotoxicity effect of TMZ is reached by DNA alkylation on $\mathrm{O}^{6}$ position of guanine and $\mathrm{N}^{3}$ position of adenine. During DNA replication, methylated guanine will couple with thymidine (which should be coupled with cytidine) thus cause mismatch repair and induce apoptosis. ${ }^{5,7}$ These cellular repair mechanisms are dysfunctional and lead to apoptosis. ${ }^{8}$ Bioavailability of oral TMZ is $100 \%$ and possesses good penetration through blood-brain barrier as well as brain tumor tissue. TMZ is well tolerated. Mostly occurred adverse drug reactions are nausea, vomiting, constipation, diarrhea, thrombocytopenia, neutropenia, and anemia.

In phase III multicenter randomized clinical trial EORTCNCIC, a patient with newly diagnosed glioblastoma 
randomly assigned to radiotherapy and TMZ dosage 75 $\mathrm{mg} / \mathrm{m}^{2}$ daily followed by 6 cycles adjuvant TMZ 150-200 $\mathrm{mg} / \mathrm{m}^{2}$ for day $1-5$ in each 28 -day cycle. The result showed that average survival rate in this combination treatment group significantly increased 14,6 months as compared to radiation alone (12.1 months). Patient's quality of life was not significantly reduced except the elevation of fatigue. ${ }^{7}$

\section{Monitoring of Adverse Drug Reactions}

Almost all conventional chemotherapeutic agents modulate cell proliferation process. As cell proliferation is the primary biological process in all cell type include normal cell, chemotherapeutic agents will also influence the normal biological process and induce adverse drug reactions. ${ }^{6}$ Adverse drug reactions in the neurological field are related to neurotoxicity which is occurred in the early or late stage from the starting dose of chemotherapy. This side effect could affect the treatment planning for a specific patient. In general, the incidence of neurotoxicity related to chemotherapeutic agents is unknown; however, these particular side effect is related to elevation of dosage of new agents and the usage of growth factor and/ or blood stem cells to prevent myelosuppression. Moreover, as the number of survivor increase, neurological side effect mainly present at late response such as cognitive impairment and/ or dementia was increased. ${ }^{3}$

Overall, there is none of the well established universal system used to determine the degree for the evaluation of neurological toxicity in a patient treated with chemotherapeutic agents. However, there is two mostly used scoring system for this purpose: NCI-CTC 3 (National Cancer Institute Common Toxicity Criteria version 3) and ECOG (Eastern Cooperative Oncology Group). Patient with a toxicity score of 1 or 2 is suggested to continue treatment while a patient with a toxicity score of 3 or 4 will require dosage modification or treatment discontinuation. ${ }^{3}$

Table 1. Clinical manifestation of peripheral and central nervous system chemotherapy-related side effects in glioma patients.

\begin{tabular}{ll}
\hline Clinical Manifestations & Symptoms \\
\hline Cognitive impairment & $\begin{array}{l}\text { Reduced cognitive function in } \\
\text { all domains include working } \\
\text { (Chemobrain) } \\
\text { memory, executive function, } \\
\text { and processing time }\end{array}$
\end{tabular}

Acute encephalopathy

Leukoencephalopathy

Cerebellar dysfunction

Spinal cor toxicity

Peripheral neuropathy
Lethargy, confusion, somnolence, convulsion, and coma

Cognitive impairment which could be developed into dementia, coma, and death

Cerebellar dysfunction include truncal ataxia, gait disorders, and ataxia

Spinal cord and radicular symptoms, Lhermitte sign

Acute pain syndrome, paresthesia, sensory ataxia, and mechanical and cold allodynia

\section{Adverse Drug Reactions on Central Nervous System Cognitive Impairment (Chemobrain)}

Cognitive change associated with chemotherapy was reported in 1970, but the first systematic study was conducted around 1990. Cognitive disorders related to chemotherapy usually characterized by mild symptoms (reduced function within reasonable limit) and occurred at various cognition domain involving working memory, executive function, and processing time. Although acute cognitive alterations are common, only a few of these disorders present in the long term in case of cancer patients (17-34\%). ${ }^{9}$ The traditional understanding underlying these disorders remains unknown and varied depends on the type of chemotherapy used for the patients. ${ }^{10}$ Previously, these disorders are suggested to be correlated with psychological factors such as depression or anxiety, or other chemotherapy-related side effects such as fatigue; but recent studies demonstrated that after this factor had been controlled, cognitive alteration still persistently occurred. ${ }^{11}$ "Chemobrain" is well-known terminology for describing cognitive function alterations reflected the toxic effects of systemic chemotherapy on the central nervous system. This condition is increasingly studied within the last 10 years because the number of survivors was being elevated as technology developed. ${ }^{6}$

Chemotherapy also causes alteration on structural and functional of brain tissue which could be found on imaging examination. In chemotherapy-treated patient, MRI showed reduced brain volume specifically at the essential part for cognitive function (e.g. frontal cortex) and altered integrity of tractus substansia alba which connect the brain structure and also reduced activity in the frontal area which is important for working memory. Furthermore, there is a reduction of substantia grisea and substantia alba volume. ${ }^{6}$ EEG examination showed decreased amplitude (intensity of neuronal activity) and latency (time and duration of activation) related to the capacity for processing information. ${ }^{9}$

Almost all chemotherapeutic drugs potentially trigger central nervous system disorder such as encephalopathy, leukoencephalopathy, ototoxicity, and cerebellar symptoms, although this condition rarely occurs. Assessment with positron emission tomography (PET) revealed that radiolabelled cisplatin, BCNU (1,3-bis (2-chloroethyl)-1nitrosourea or carmustine) and paclitaxel was found at low level in brain tissue. In a rodent model, chemotherapy was associated with increased cell death and reduced cell division in the subventricular zone, gyrus dentata hippocampus, and corpus callosum which are essential for cognitive function, and for several cases, this pattern is present for several weeks after discontinuation. Neuronal progenitor cell and oligodendrocyte are fragile. These findings were seen in low dose treatment compared to the dosage required for killing tumor cells. ${ }^{9}$

Factors that might affect chemotherapeutic agents distribution into central nervous system is a genetic variation of drugs transporter in the blood-brain barrier. Multidrug resistance 1 (MDR 1) gene encodes $\mathrm{P}$ glycoprotein $(\mathrm{P}-\mathrm{gp})$ protein which protects cells from noxious substances by transporting those substances into extracellular space. P-gp was expressed in capillary endothelial cells in the brain. In the blood-brain barrier, Pgp influences the number of drug's uptake into brain tissue. 
Polymorphism on MDR1 gene was demonstrated to affect P-gp functionality. One of polymorphism type of P-gp protein which has been widely investigated is polymorphism at $\mathrm{C} 3435 \mathrm{~T}$ exon 26 , causing more drugs to enter or less drug being pumped out from brain tissue. Patient with less expressed or reduced activity of P-gp is vulnerable to the long term cognitive side effect because of excessive exposure of chemotherapy in their brain tissues. ${ }^{9}$

Besides genetic variation, oxidative stress caused by exogenous toxin is considered to be related to this condition. Several chemotherapeutic agents (e.g. alkylating agents) reach their therapeutic effect by destructing DNA thus lead to apoptosis. However, DNA in normal cells is also influenced and trigger adverse drug reactions. Chemotherapy is correlated with elevation of free iron and free radicals, decreased antioxidant capacity, and those conditions will cause oxidative stress and DNA damage. ${ }^{9}$

Basic understanding of DNA damage in central nervous system caused by chemotherapy is not well understood. The central nervous system might be vulnerable to elevated oxidative stress because the increased level of endogenous metabolite and the ROS production. Moreover, reduced capability to repair damaging mitochondrial DNA also associated with apoptosis. Damage of DNA also inhibit the transcription and cause loss of important gene expression. Besides its effect on DNA, chemotherapy could affect the length of the telomere. During DNA replication, the telomere is shrunk 20-200 base pair for each replication process. Telomere shrinkage into a certain level will cause cell aging and apoptosis. ${ }^{9}$

The relationship between chemotherapy and cognitive alteration is proved by a previous study using paclitaxel and docetaxel. These drugs administration was associated with an increased level of IL6, IL8, and IL10. However, there is no strong evidence that showed a direct association between cognitive alteration and cytokine deregulation. Cognitive alteration might be correlated with cytokine deregulation both in a direct or indirect manner. Neuronal damage caused by cytokine exposure could result from several mechanisms such as cytotoxicity related to glutamate receptor and oxidative stress. Indirect effect involve decreased appetite followed with micronutrient deficiency and sleep disorder. ${ }^{9}$

The other factors are related to estrogen and testosterone hormone. The previous study demonstrated that estrogen and testosterone possessed neuroprotection and antioxidant capacity, and estrogen also maintains the length of the telomere. Reduced activity of estrogen and testosterone might independently influence cognitive function or interact with chemotherapy through the reduction of antioxidant capacity or capability of maintaining the length of telomere. ${ }^{9}$

Genetic susceptibility is determined by apolipoprotein E (APOE), a complex glycoprotein, which facilitates uptake, transport, and distribution of fat, and has an essential role on neuronal repair and plasticity post injury and catecholO-methyltransferase (COMT). ${ }^{9,11}$ There are three exons gene encoding APOE in chromosome 19 in human and 3 main alleles: E2, E3, and E4. E4 allele is associated with several diseases characterized by prominent cognitive impairment such as a normal patient with memory impairment, Alzheimer, and poor outcome in a patient with stroke and traumatic brain injury. E4 carrier showed different morphology particularly the volume of the bottom part of the hippocampus (suggested a reduced functional capacity reflecting important memory function) compared with another allele of APOE. ${ }^{9}$ COMT affects the metabolism of catecholamine via dopamine methylation. Patient with allele Val demonstrated a low level of dopamine in their frontal cortex caused by faster dopamine metabolism compared with allele Met. The researcher found that patient with breast cancer who had combined allele of COMT and treated with chemotherapy, showed bad attention, verbal fluency, and motoric activity as compared to homozygote COMT-Met. ${ }^{11}$

Several factors associated with increased risk of chemotherapy-related neurotoxicity are (1) high dose exposure of chemotherapeutic regiments or elevated concentration of its metabolite caused by impaired clearance and/ or impaired pharmacogenetics modulation of drug's pharmacokinetics; (2) addition or synergy effect of several chemotherapeutic agents; (3) addition or synergy effect of multimodal treatment such as chemotherapy and radiation; (4) intra-arterial administration cause destruction of blood-brain barrier; (5) intrathecal administration. However, the previous study still could not discover specific predictor to determine the potential increase of chemotherapy-related cognitive impairment. ${ }^{12}$

Nokia and colleagues in 2012 conducted study investigated the adverse drug reaction on rodent model injected with TMZ for several weeks. In human, common dosage of TMZ is $75-200 \mathrm{mg} / \mathrm{m}^{2}$ or approximately $2-5 \mathrm{mg} / \mathrm{kg}$. The rat was injected once daily for 3 days followed by 4 days recovery and performed for 6 weeks. This protocol is similar to protocol performed in human $\left(200 \mathrm{mg} / \mathrm{m}^{2}\right.$ per day for 5 days every 4 weeks). The result showed that chemotherapy reduces neurogenesis and endogenous theta activities disrupt the learning process and response of thetaband toward stimulus. This TMZ effect is occurred within several weeks after treatment and only affected by stimulus requiring association across the temporal gap and will not occur in exercise with overlap stimulus. Chemotherapy did not alter the memory of previously learned association, a memory which did not depend on the new hippocampal neuron. $^{13}$

IN TMZ-treated rats, evidence showed a reduction of new cells in the granule cell layer in hippocampus approximately $34 \%$. This finding suggests that TMZ may reduce proliferated cell population and in the other hand increase the number of died post-mitotic cells. Chemotherapy effect on neurogenesis is not only restricted to drugs penetrating blood-brain barrier but also alter immature cells differentiation or migration during treatment. TMZ affect neurogenesis more significant compared to the glial formation (in the hilar region), and also cells which are possible to become neuron (in granule cell layer). Chemotherapy selectively impaired learning process which is learning process originated from mature neuronal cells in cerebellum and memory from mature neuronal cells in neocortex still intact. Chemotherapy did not affect retention or memory expression process. ${ }^{13}$

In human, temozolomide administration as an adjuvant for radiotherapy is become standardized treatment and increase the 2-year survival rate 2-fold as compared to radiation treatment only. Long term follow up showed a little 
evidence about additional toxicity effect or synergism from this regiments. ${ }^{10}$

\section{Acute Encephalopathy}

Acute encephalopathy is a common condition found in a cancer patient, with various precipitation factors such as metabolic, hypoxia, brain metastases, meningeal carcinomatosis, infection, paraneoplastic phenomenon, and drugs. Symptoms related to this disease are lethargy, confusion, somnolence, convulsion, and coma. In druginduced encephalopathy, this condition should be differentiated with other situation by detailed history taking include narcotics-analgesic medication and anti-emetic which are commonly used in a patient treated with chemotherapy. Central nervous system infection should be excluded especially in immunocompromised and neutropenic patients. Laboratory examination required for this condition consists of urea/ electrolyte, liver function, serum glucose, magnesium, calcium, virus serology, and cerebrospinal fluid examination. If a focal neurological deficit is found, CT scan and MRI could be performed as additional workup, and for a particular case. This imaging modality is performed before lumbar puncture. EEG can be conducted in a patient with seizure, and EEG examination usually shows general deceleration mainly characterized by delta activity. Cytotoxic drugs-induced encephalopathy is usually a self-limiting disease and spontaneously healed. The only specific treatment for drug-induced encephalopathy is methylene blue for ifosfamide-induced encephalopathy and should be considered for all patient treated with ifosfamide. Risk factor for the development of this condition include extreme age, dose/ schedule of regiments, previous cranial radiotherapy, and renal or hepatic impairment. ${ }^{3}$

\section{Leukoencephalopathy}

Leukoencephalopathy is a disorder of substantia nigra caused by both endogenous or exogenous factor, including chemotherapy. ${ }^{14}$ This condition could happen after acute encephalopathy, but also present as the first symptom of neurotoxicity after several months or years chemotherapy treatment. $^{3}$ Neurologic symptoms could be varied. ${ }^{14}$ Patients usually come with cognitive dysfunction which could extend into dementia, coma, and death. MRI imaging usually showed extensive alterations in substantia alba. Histologically, there are an axonal swelling, demyelination, and neuronal death. Patient with a higher risk of this condition is patient treated with methotrexate or cytarabine is especially given as intrathecal regiment or previous cranial radiotherapy such as in a patient with lymphoma of CNS. Until now, there is no specific treatment for preventing its progressivity thus it has poor prognosis. ${ }^{3}$

\section{Cerebellar Dysfunction}

Cerebellar symptoms are mostly present because of direct spreading of cancer especially in the asymmetric tumor, but this condition also found in a paraneoplastic syndrome which has a subacute effect and could be associated with the anti-neuronal antibody. Cerebellar ataxia should be distinguished from sensory ataxia caused by sensory neuropathy. Cytarabine and 5-fluorouracil are the most common used cytotoxic drugs causing cerebellar dysfunction characterized by truncal ataxia, gait disorders, and ataxia. MRI imaging might show normal results, but serial examination could identify chronic atrophy triggered by the irreversible loss of Purkinje cells. ${ }^{3}$

\section{Spinal Medulla Toxicity}

Spinal cord toxicity usually occurred after intrathecal administration of cytotoxic drugs for acute leukemia, lymphoma, and brain tumor. Intrathecal administration of brain tumor is given as part of lumbar puncture procedure or directly into ventricle via reservoir Ommaya. Common medicines used in this condition are cytarabine, methotrexate, and hydrocortisone, which could be administered as single regiments or in combination. Symptoms represented this condition and usually occurred after several cycles of treatments include spinal cord symptoms and radicular symptoms. Several cases reported some symptoms as Lhermitte's sign. The decreased neuronal function could develop in an upward direction. Histologically, this toxicity is reflected by focal necrosis area, especially in peripheral medulla spinalis which is linked to axonal swelling and demyelination. Cerebrospinal fluid usually revealed an increased level of myelin basic protein. Evidence showed only half of the patient followed by improvement.

\section{Adverse Drug Reactions on the Peripheral Nervous System}

\section{Peripheral Neuropathy}

Chemotherapy-induced peripheral neuropathic pain (CIPNP) is one of the severe adverse drug reactions of cytotoxic drugs. CIPNP incidence requires dosage adjustment, drug type selection, delay of treatment until the next cycle. Peripheral neuropathy is reflected by several symptoms pain immediately occurred after cytotoxic administration such as paclitaxel acute pain syndrome (PAPS), until paresthesia, sensory ataxia, and mechanical and cold allodynia. The part that mostly affected is extremity, but repeated exposure could induce symptoms at another part of the body. Substances that could induce CIPNPN are taxanes, platinum derivate, and vinca alkaloid, and rarely ixabepilone, bortezomib, and thalidomide. ${ }^{15}$ The severity of neuronal damage depends on various factors such as the type of chemotherapy, duration of therapy, cumulative dose, and usage of other neurotoxic drugs. Patient's factors such as age and condition lead to neuronal damage such as diabetes and alcohol administration are important as well. Furthermore, a neuron-related condition also affects neuronal function susceptibility to drug-induced neurotoxicity and lead to symptomatic neuropathy. Instead of an improvement of their symptoms, a patient with a history of CIPN is considered to have a higher risk to undergo progressive neuropathy if they are exposed to repeated exposure of neurotoxic drugs. ${ }^{16}$

Pathophysiology of CIPNP is not well understood and could be different depending on drug classes used. In general, chemotherapy drugs cause peripheral neuron damage through destruction of microtubule and alteration of microtubule's axonal transport, by damaging mitochondria and its cytotoxic effect on DNA. ${ }^{16}$

CIPNP is also correlated with the primary sensory neuron in dorsal root ganglia (DRG). Paclitaxel, oxaliplatin, and vincristine have a harmful effect on DRG neuron. These substances induce the activity of voltage-gated ion channels such as sodium channel $\left(\mathrm{Na}_{\mathrm{v}}\right)$ and ligand-gated ion channel such as calcium channel $\left(\mathrm{Ca}_{\mathrm{v}}\right)$, transient receptor potential 
(TRP) channel and produce reactive oxygen species (ROS) that disrupt mitochondria's electron transport chain thus alter ATP production in a sensory neuron. Furthermore, neuropathy induced by paclitaxel or vincristine involved strong inflammatory component which is less toxic compared with oxaliplatin-induced CIPNP. An inflammatory component in this situation was resulted from activation of microglia, astrocyte, and satellite glial cells within cornu dorsalis of the spinal cord which in turn induce production and release of proalgesia mediators such as tumor necrosis factor (TNF) and IL-1 $\beta .^{15}$

Methods used to evaluate the severity/ grade of CIPNP in clinical studies is not homogenous. However, most clinical studies used scoring from the National Cancer InstituteCommon Toxicity Criteria (NCI-CTC) and Total Neuropathy Score (TNS) which is designed for evaluating the severity of distal polyneuropathy. ${ }^{17}$

In the context of prevention of CIPNP in a patient received chemotherapy drugs, we have to consider that patient has already exposed to a various combination of drugs and each drug has its potential side effects. Therefore, more drugs administered to the patient will potentially induce crossreaction or interaction and also affect the efficacy of cytostatic therapy. This rational approach leads to a recommendation for giving monotherapy in a patient with CIPNP. Moreover, antioxidant and neuroprotective drugs could be administered to overcome the damage related to ROS and other oxidant products. Choosing an appropriate antioxidant is complicated because each antioxidant has a specific effect on cells and tissues and its distribution are dependent on the physicochemical properties such as lipid solubility. Recently, there is no strong clinical evidence showed the efficacy of antioxidants on CIPNP. However, a small randomized clinical trial reported that administration of endogenous lipophilic antioxidants $\alpha$-tocopherol (vitamin E) dosage 300-600 mg/day and $800 \mathrm{mg} /$ day had no protective effect on CIPNP. On the other hand, another double-blind, a randomized clinical trial reported that administration of omega-3 fatty acid and eicopentaenoic acid $640 \mathrm{mg} /$ day (3 times per day) showed a beneficial effect on paclitaxel-induced neuropathy. Both substances had been known for its neuroprotective, anti-inflammatory, and anti-nociceptive effect. ${ }^{15}$

Drug of choice for neuroprotective effect on platinumbased chemotherapy such as cisplatin/ carboplatin had been widely investigated. Review study conducted by Cochrane Library reported that data about neuroprotective effect several substances or drugs such as amifostine, diethyldithiocarbamate, glutathione, Org 2766 and vitamin E is not enough to support neuroprotective effect on cisplatininduced peripheral neuropathy. In a patient receiving cisplatin-based chemotherapy, polyneuropathy could occur at dosage $250-350 \mathrm{mg} / \mathrm{m}^{2}$ and almost patient experience polyneuropathy at cumulative dose $500-600 \mathrm{mg} / \mathrm{m}^{2}$. Therefore, consideration of dosage adjustment or frequency adjustment is required in the case of polyneuropathy. In vincristine-induced neuropathy (classified as vinca alkaloids) is reversible as chemotherapy discontinued. Duration of paresthesia and motoric weakness usually improved after 3 months without chemotherapy. To minimize the neurotoxic effect of vincristine, vincristine is recommended at dose $1,4 \mathrm{mg} / \mathrm{m}^{2}$ every single dose with maximal dose at $2 \mathrm{mg}$ in a single dose without calculating the body surface. There is no pharmacological therapy could be used to decrease or prevent vinca alkaloid-induced peripheral neuropathy. ${ }^{17}$

Table 2. Clinical symptoms CIPN and classification of drugs. ${ }^{18}$

\begin{tabular}{|c|c|}
\hline $\begin{array}{l}\text { Chemotherapeutic } \\
\text { drugs }\end{array}$ & Clinical symptoms \\
\hline Cisplatin & $\begin{array}{l}\text { Paresthesia, numbness with stocking } \\
\text { and glove distribution }\end{array}$ \\
\hline Oxaliplatin & $\begin{array}{l}\text { Paresthesia, numbness and/ or } \\
\text { neuropathic pain stocking and glove } \\
\text { distribution, neuromyotonia-like } \\
\text { symptoms }\end{array}$ \\
\hline Paclitaxel & $\begin{array}{l}\text { Paresthesia, numbness and/ or } \\
\text { neuropathic pain stocking and glove } \\
\text { distribution, myalgia, myopathy }\end{array}$ \\
\hline Docetaxel & $\begin{array}{l}\text { Paresthesia, numbness and/ or } \\
\text { neuropathic pain stocking and glove } \\
\text { distribution, myalgia, myopathy }\end{array}$ \\
\hline Epothilones & $\begin{array}{l}\text { Paresthesia, numbness and/ or } \\
\text { neuropathic pain stocking and glove } \\
\text { distribution, myalgia, myopathy }\end{array}$ \\
\hline Bortezomib & $\begin{array}{l}\text { Neuropathic pain and paresthesia at } \\
\text { distal extremities }\end{array}$ \\
\hline Thalidomide & $\begin{array}{l}\text { Neuropathic pain and paresthesia at } \\
\text { distal extremities }\end{array}$ \\
\hline Lenalidomide & $\begin{array}{l}\text { Neuropathic pain and paresthesia at } \\
\text { distal extremities }\end{array}$ \\
\hline Pomalidomide & $\begin{array}{l}\text { Neuropathic pain and paresthesia at } \\
\text { distal extremities }\end{array}$ \\
\hline Vincristine & $\begin{array}{l}\text { Paresthesia, numbness and/ or } \\
\text { neuropathic pain stocking and glove } \\
\text { distribution, muscle cramp, mild distal } \\
\text { motoric weaknesses }\end{array}$ \\
\hline Suramin & $\begin{array}{l}\text { Paresthesia and hyperesthesia with } \\
\text { symmetrical/ bilateral pain, particularly } \\
\text { in the distal region }\end{array}$ \\
\hline
\end{tabular}

In taxane-induced polyneuropathy (e.g., paclitaxel, docetaxel), the symptoms will be relieved or improved after 3-6 months drug's discontinuation. However, in some circumstances, severe symptoms could persist in a long time after discontinuation. Neuroprotective drugs administration such as thiol, neurotrophic factors, and antioxidants were not proved in a clinical trial setting. Administration of glutamine, acetyl-L-carnitine and vitamin E $600 \mathrm{mg}$ twice a day has neuroprotective effects in this situation but still in investigation. ${ }^{17}$

\section{Conclusion}

Common side effects of chemotherapy on the central nervous system are chemobrain, acute encephalopathy, leukoencephalopathy, cerebellar dysfunction, spinal cord toxicity. Chemotherapy also possesses toxicity on the peripheral nervous system which is termed as Chemotherapy-induced peripheral neuropathic pain (CIPNP). CIPNP usually affected by dose and subclasses of drugs. CIPNP is usually treated by adjustment of dosage; prolong the interval of drug administration. CIPNP is a reversible condition thus the symptoms usually relieve after several months of discontinuation. In the clinical setting, recognition of chemotherapy-induced neurotoxicity is essential to improve patient's quality of life with glioma. 


\section{Acknowledgement}

The authors would like to thank to Brawijaya University and Saiful Anwar General Hospital, Malang, East Java, Indonesia for facilitating this review.

\section{References}

1. Lapointe, S, Perry A, Butowski NA. Primary brain tumours in adults. The Lancet; 2018.392(10145),432446. DOI: 10.1016/s0140-6736 (18)30990-5

2. Westphal M, Lamszus K. The neurobiology of gliomas: from cell biology to the development of therapeutic approaches; 2011.12(September):495-508. DOI: $10.1038 /$ nrn3060

3. Rees JH, Wen PY. Blue books of neurology. philadelphia: Elsevier; 2010.p. 352-371

4. Ostrom QT, Bauchet L, Davis FG, Deltour I, Fisher JL, Langer CE, et al. The epidemiology of glioma in adults: A state of the science review. Neuro Oncol; 2014.16(7):896-913. DOI: 10.1093/neuonc/nou087

5. Parisi S, Corsa P, Raguso A, Perrone A, Cossa S, Munafò $\mathrm{T}$, et al. Temozolomide and radiotherapy versus radiotherapy alone in high grade gliomas: a very long term comparative study and literature review. BioMed Research International; 2015.1-7. DOI: $10.1155 / 2015 / 620643$

6. Simó M, Rifà-ros X, Rodriguez-fornells A, Bruna J. Chemobrain: A systematic review of structural and functional neuroimaging studies. Neurosci Biobehav Rev; 2013.1-11. DOI: http://dx.doi.org/10.1016/j.neubiorev.2013.04.015

7. Michaud K, Chang SM. Principles of chemotherapy in youmans neurological surgery. in: sixth edit. Saunders; 2011. p. 1236-42

8. Wesolowski JR, Rajdev P, Mukherji SK. Temozolomide (Temodar). Am J Neuroradiol; 2010.31(8):1383-4. DOI: 10.3174/ajnr.A2170

9. Ahles TA, Saykin AJ. Candidate mechanisms for chemotherapy-induced cognitive changes. Nat Rev Cancer; 2007.7(3):192-201. DOI: 10.1038/nrc2073
10. Abrey LE. The impact of chemotherapy on cognitive outcomes in adults with primary brain tumors. 2012.285-90. DOI: 10.1007/s11060-012-0807-6

11. Ahles TA, Root JC, Ryan EL. Journal of clinical oncology cancer and cancer treatment - associated cognitive change : an update on the state of the science; 2015.30(30). DOI: 10.1200/JCO.2012.43.0116

12. Schagen SB, Wefel JS. Chemotherapy-related changes in cognitive functioning. Ejc Suppl; 2016.11(2):22532.

DOI: http://dx.doi.org/10.1016/j.ejcsup.2013.07.007

13. Nokia MS, Anderson ML, Shors TJ. Chemotherapy disrupts learning, neurogenesis and theta activity in the adult brain; 2012.36(May):3521-30. DOI: 10.1111/ejn. 12007

14. Sindhwani G, Arora M, Thakker VD, Jain A. MRI in chemotherapy induced leukoencephalopathy: report of two cases and radiologist's perspective. J Clin Diagn Res; 2017.11(7): TD08-TD09. DOI: 10.7860/JCDR/2017/29164.10248

15. Sisignano M, Baron R, Scholich K, Geisslinger G. Mechanism-based treatment for chemotherapy-induced peripheral neuropathic pain. Nat Rev Neurol; 2014.10(12):694-707.

10.1038/nrneurol.2014.211

16. Sarosiek KA, Ni Chonghaile T, Letai A. Mitochondria: gatekeepers of response to chemotherapy. Trends in Cell Biology; 2013. 23(12), 612-619. DOI: 10.1016/j.tcb.2013.08.003

17. Argyriou AA, Bruna J, Marmiroli P, Cavaletti G. Chemotherapy-induced peripheral neurotoxicity (CIPN): An update. Crit Rev Oncol/Hematol; 2012.82(1):51-77. DOI: http://dx.doi.org/10.1016/j.critrevonc.2011.04.012

18. Argyriou AA, Kyritsis AP, Makatsoris T, Kalofonos HP. Chemotherapy-induced peripheral neuropathy in adults: a comprehensive update of the literature. Cancer Manag; 2014.6, 135-147 DOI: 10.2147/CMAR.S44261 